
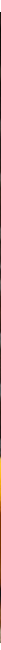

Neues Konzept für die COPD

\title{
Treatable Traits sollen exakte Diagnose ersetzen
}

\begin{abstract}
Für einen strategischen Wandel im Umgang mit der COPD plädiert Prof. Alvar Agusti, Barcelona: Künftig sollten behandelbare Charakteristika („treatable traits“) des einzelnen Patienten identifiziert und gezielt angegangen werden.
\end{abstract}

Grundlage des neuen COPD-Konzepts ist die Überlegung, dass die chronisch-obstruktive Lungenerkrankung keine einheitliche Erkrankung darstellt, sondern Oberbegriff für ein ausgesprochen komplexes und heterogenes Spektrum von Krankheiten ist. „Zuerst müssen wir definieren, was wir überhaupt behandeln wollen - Exazerbationen? Symptome? Begleiterkrankungen?", sagte Prof. Alvar Agusti, Direktor der Thoraxklinik am Universitätsklinikum Barcelona.

Statt die Therapie wie bisher primär am Phänotyp, also an der Symptomatik auszurichten, wird man sich künftig stärker am Endotyp orientieren, sprich Biomarker nutzen, um die Therapie zu steuern, prophezeite Agusti. Die GOLD-Gruppe (Global Initiative on Chronic-Obstructive Lung Disease) sei schon auf dem richtigen Weg, weil sie nicht mehr allein die Lungenfunktion in Patientenklassifikation und Therapieplanung einbezieht, sondern auch Symptome und Exazerbationsrisiko. Das reiche aber noch nicht aus.

\section{Patientenmanagement soll individueller werden}

Gemeinsam mit elf anderen GOLD-Mitgliedern, darunter aus Deutschland Prof.Claus Vogelmeier, Chef der Pneumologie am Universitätsklinikum Marburg, hat Agusti das Konzept der „treatable traits" entwickelt, das ein noch individuelleres Patientenmanagement erlauben soll. Denn im klinischen Alltag lassen sich Patienten meist nicht so sauber einteilen, wie GOLD dies vorsieht: „Das Vorliegen eines Phänotyps schließt einen zweiten nicht aus", betonte Agusti.

Im Einzelfall sähe das dann so aus: Bei einem Erwachsenen mit entsprechenden Symptomen besteht das Minimalprogramm nach Anamnese und klinischer Untersuchung aus Spi- rometrie, der Bestimmung von NO im Exhalat und der Eosinophilen im Blut. Ergibt sich daraus ein starker Hinweis auf eine chronische Atemwegserkrankung, werden die behandelbaren Charakteristika identifiziert - wohlgemerkt, ohne Umweg über die Ausstellung einer exakten Diagnose - und therapiert.

Die Obstruktion aufgrund eines erhöhten Muskeltonus in den Atemwegen wäre also ein Fall für potente Bronchodilatatoren, bei einer eosinophilen Entzündung fänden beispielsweise inhalative Steroide ihren Platz und bei einer bakteriellen Kolonisation Antibiotika. Bisher gibt es drei Listen mit treatable traits - pulmonale, extrapulmonale sowie Lebensstil/ Verhalten -, die aber natürlich jederzeit ergänzt werden können. Diagnostik wird also nicht überflüssig, aber die strategische Ausrichtung ändert sich.

\section{Fallen die Grenzen zwischen Asthma und COPD?}

Natürlich können - und werden wahrscheinlich meistens mehrere treatable traits im selben Patienten koexistieren. Der Charme des Konzepts liegt darin, dass man anschaut, was da ist und das behandelt, ohne sich lange mit der Differenzialdiagnose aufzuhalten. „Wir stellen tatsächlich auch die Frage, welche Bedeutung die Unterscheidung von Asthma und COPD noch hat", meinte Agusti.

Er räumte zugleich ein, dass es sich hierbei nicht um ein evaluiertes und validiertes Konzept handelt, sondern um einen Vorschlag, der eine Diskussion in Gang bringen soll. „Wir glauben, dass die Treatable-traits-Strategie die Patientenversorgung verbessern kann." 